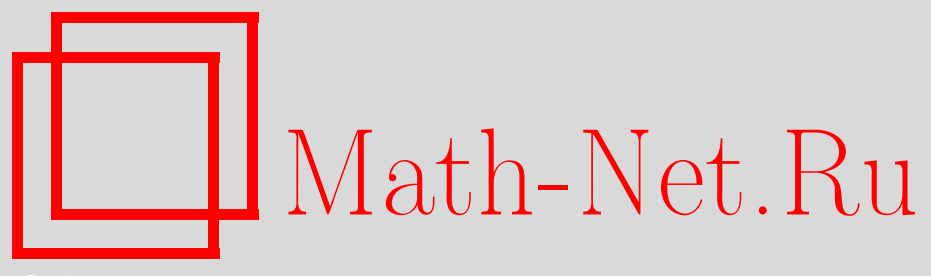

Э. Р. Бальзин, Производные сечения, факторизационные алгебры и гипотеза Делиня, Матем. заметки, 2016, том 100, выпуск 2, 291-295

DOI: https://doi.org/10.4213/mzm11202

Использование Общероссийского математического портала Math-Net.Ru подразумевает, что вы прочитали и согласны с пользовательским соглашением http://www.mathnet.ru/rus/agreement

Параметры загрузки:

IP: 54.147 .182 .235

26 апреля 2023 г., 15:31:31

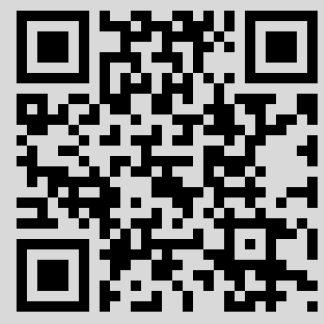




\section{Производные сечения, факторизационные алгебры и гипотеза Делиня}

\section{Э. Р. Бальзин}

Ключевые слова: гомотопическая алгебра, модельные категории, модельные структуры Риди расслоения Гротендика, факторизационные алгебры, операторные категории, гипотеза Делиня.

DOI: $10.4213 / \mathrm{mzm} 11202$

Задача по описанию мультипликативной структуры кратных пространств петель активно [1] изучалась в семидесятые годы прошлого века. Решение этой задачи привело к возникновению двух формализмов: подхода операд [2], а также формализма Сигала [3].

ОПРеДЕЛЕНИЕ 1. Операдой $\mathscr{O}$ в симметрической моноидальной категории [4] (далее $\mathrm{CMK}) \mathscr{M}$ называется набор объектов $\mathscr{O}(n), n \in \mathbb{N}$, с действием симметрической группы $\Sigma_{n}$ и заданными законами композиции $\mathscr{O}(n) \otimes \mathscr{O}(k) \rightarrow \mathscr{O}(n+k-1)$, которые ассоциативны, совместимы с действием симметрических групп и обладают единицей (подробное определение дано в [5]). Структурой $\mathscr{O}$-алгебры на объекте $X$ называется семейство совместимых со структурой $\mathscr{O}$ отображений $\mathscr{O}(n) \otimes X^{\otimes n} \rightarrow X$.

ПримеР 2. В категории Тор топологических пространств с декартовым умножением существует такая операда $\mathrm{E}_{n}$, где $n \in[1, \infty]$, что всякое $n$-кратное пространство петель $\Omega^{n} Y$ является $\mathrm{E}_{n}$-алгеброй.

Отметим, что алгебры над операдой имеет смысл рассматривать только с точностью до готомопической эквивалентности. Обычная техника для этого - гомотопическая алгебра и модельные категории в смысле работы Квиллена [6]-[8]. Это же верно для операд; например, в качестве $\mathrm{E}_{\infty}$-операды годится любая операда $\mathscr{O}$ такая, что всякое $\mathscr{O}(k)$ стягиваемо со свободным действием $\Sigma_{k}$. В качестве $\mathrm{E}_{n}$-операды для конечного $n$ можно взять такую, что $\mathrm{E}_{n}(k)$ гомотопически эквивалентно пространству $k$ различных точек в $n$-мерном диске.

Вместо Top можно взять моноидальную категорию DVect $_{k}$ комплексов векторных пространств с тензорным произведением и рассмотреть в ней операды $\mathbb{E}_{n}$, полученные взятием цепных комплексов от операд $\mathrm{E}_{n}$ из примера 2. Одно из утверждений, которое можно доказать в DVect $_{k}$ - известная

Теорема 3 (гипотеза Делиня [9]-[11]). Пусть $A-\mathrm{dg}$-алгебра и $C H(A)-$ ее когомологический комплекс Хохшильда. Тогда на $C H(A)$ дейстует операда $\mathbb{E}_{2}$.

Доказательства этого утверждения нетривиальны, и их нетривиальность связана с выбором операды: квазиизоморфные операды, описывающие эквивалентные алгебраические структуры на когомологиях, могут сильно отличаться по своей сложности.

Опишем теперь подход Сигала. В [3] рассматриваются функторы $X: \Gamma \rightarrow$ Top, где $\Gamma-$ категория отмеченных конечных множеств, со специальными условиями. В формализме операд таким структурам соответствуют алгебры над операдой $\mathrm{E}_{\infty}$. Функторы, подобные $X$, задают наборы диаграмм вида

$$
A^{n} \leftarrow B(n) \rightarrow A
$$

и специальное условие на $X$ состоит в том, что левая стрелка в (1) является гомотопической эквивалентностью. Не обязательно при этом, что $B(n) \cong \mathrm{E}_{\infty}(n) \times A^{n}$. Формализм Сигала не имеет проблем, связанных с выбором модели для операды $\mathrm{E}_{\infty}$, и может быть обобщен на другие алгебраические структуры посредством замены Г на другие операторные [12] категории. Однако, в существующем виде он применим только к СМК, произведение в которых

DOI: $10.4213 / \mathrm{mzm} 11202$ 
декартово. Тензорное произведение комплексов не является декартовым, а потому подход Сигала к DVect $_{k}$ требует модифицирования.

Например, это можно осуществить следующим образом. Произведение в категории Cat малых категорий декартово, а потому формализм Сигала применим в этом случае.

Конструкция 4. Всякая СМК $\mathscr{M}$ отвечает функтору $F: \Gamma \rightarrow$ Cat со значением $F(S) \cong$ $\mathscr{M}^{S \backslash s}$, где $s \in S$ - отмеченная точка. Эквивалентное описание таких функторов [13] формализм (оп)-расслоений Гротендика. В рамках этого языка СMK $\mathscr{M}$ отвечает функтор [14], [3] $p: \mathscr{M}^{\otimes} \rightarrow$ Г такой, что слои $\mathscr{M}^{\otimes}(S):=p^{-1}(S) \mathscr{M}^{S \backslash s}$ и для каждого объекта $X$ с $p(X)=S$ и отображения отмеченных множеств $f: S \rightarrow T$ существует универсальный морфизм $X \rightarrow f ! X$ в $\mathcal{M}^{\otimes}$, накрывающий $f$. Такой универсальный морфизм называется декартовым.

Обозначим через $\operatorname{Alg}(\mathscr{M})$ категорию коммутативных алгебр в $\mathscr{M}$, а через $\operatorname{Sect}\left(\Gamma, \mathscr{M}^{\otimes}\right)-$ категорию сечений функтора $p: \mathscr{M}^{\otimes} \rightarrow \Gamma$.

Конструкция 5. Всякой алгебре $A \in \operatorname{Alg}(\mathscr{M})$ соответствует сечение $s(A) \in \operatorname{Sect}(\Gamma$, $\left.\mathscr{M}^{\otimes}\right)$, которое сопоставляет множеству $T$ значение $(A, \ldots, A) \in \mathscr{M}^{\otimes}(T)$. Соответствующий функтор $s: \operatorname{Alg}(\mathscr{M}) \rightarrow \operatorname{Sect}\left(\Gamma, \mathscr{M}^{\otimes}\right)$ строго полон, а его образ легко охарактеризовать [14].

$\mathrm{K}$ сожалению, в гомотопическом контексте этот подход все равно не работает. А именно, если $\mathscr{M}$, помимо моноидальной, имеет также и модельную структуру (как, например, DVect $_{k}$ с проективной модельной структурой [8]), то коммутативные алгебры необязательно являются правильным объектом для рассмотрения и не совпадают, даже с точностью до квазиизоморфизма, с $\mathbb{E}_{\infty}$-алгебрами. Таким образом, для применения подхода Сигала в гомотопической алгебре необходимо более широкое понятие, чем сечение расслоения Гротендика.

Обобщая ситуацию, введем следующее определение.

ОПределЕниЕ 6 . Модельное оп-расслоение $p: \mathscr{E} \rightarrow \mathscr{C}$ - оп-расслоение, слои которого модельные категории, а функторы перехода сохраняют слабые эквивалентности и фибрации.

Следуя [15], [16] определим производные сечения этого опрасслоения.

ОПРедЕЛЕниЕ 7. Симплиииальной заменой $\mathbb{C}$ категории $\mathscr{C}$ называется категория $\left(\int N \mathscr{C}\right)^{\circ p}$, двойственная к категории элементов [17] нерва $\mathscr{C}$. Объектом $\mathbb{C}$ является, таким образом, набор $c_{0} \rightarrow \cdots \rightarrow c_{n}$ компонируемых морфизмов в $\mathscr{C}$ произвольной длины.

В [15] показано, как из оп-рассления $p: \mathscr{E} \rightarrow \mathscr{C}$ получить расслоение $q: \mathbf{E} \rightarrow \mathbb{C}$ такое, что

$$
\mathbf{E}\left(c_{0} \rightarrow \cdots \rightarrow c_{n}\right) \cong \mathscr{E}\left(c_{n}\right) .
$$

ОПРедЕлЕниЕ 8. Категория производных предсечений PSect $(\mathscr{C}, \mathscr{E})$ модельного оп-расслоения $p: \mathscr{E} \rightarrow \mathscr{C}$ есть категория $\operatorname{Sect}(\mathbb{C}, \mathbf{E})$ сечений расслоения $q: \mathbf{E} \rightarrow \mathbb{C}$.

Неформально, производное предсечение $X$ строит по каждому морфизму $f: c \rightarrow c^{\prime}$ диаграмму в $\mathscr{E}\left(c^{\prime}\right)$ вида

$$
f_{!} X(c) \longleftarrow X(f) \longrightarrow X\left(c^{\prime}\right),
$$

где $X(c) \rightarrow f_{!} X(c)$ - декартов морфизм в $\mathscr{E}$, накрывающий $f$.

ОПРедЕлЕниЕ 9. Категория производных сечений $\operatorname{DSect}(\mathscr{C}, \mathscr{E})$ - полная подкатегория $\operatorname{PSect}(\mathscr{C}, \mathscr{E})$, состоящая из таких $X$, что левые стрелки в диаграммах типа $(2)$ - слабые эквивалентности (точное определение дано в [15]).

Таким образом, производное сечение модельного оп-расслоения $p: \mathscr{M}^{\otimes} \rightarrow \Gamma$ задает набор диаграмм

$$
A^{\otimes S} \longleftarrow B(S) \longrightarrow A,
$$

в которых левая стрелка является слабой эквивалентностью. Это соответствует обычному формализму Сигала (1). 
Teорема 10. В вышеописанной ситуации на категории предсечений $\operatorname{PSect}(\mathscr{C}, \mathscr{E})$ существует модельная структура.

Модельная структура теоремы 10 строится аналогично модельным структурам Риди [8], что представляет независимый интерес. Кроме того, она позволяет эффективно работать с категориями производных сечений. В частности, можно доказать следующий результат.

ОБозначение 11 . Обозначим через Но $\operatorname{PSect}(\mathscr{C}, \mathscr{E})$ локализацию [6], [8] категории $\operatorname{PSect}(\mathscr{C}, \mathscr{E})$ вдоль слабых эквивалентностей, и через Но DSect $(\mathscr{C}, \mathscr{E})$ - соответствующую подкатегорию производных сечений.

ОПределЕниЕ 12. Функтор $F: \mathscr{D} \rightarrow \mathscr{C}$ называется резольвентой, если для каждой последовательности стрелок $c_{0} \stackrel{f_{1}}{\longrightarrow} \cdots \stackrel{f_{n}}{\longrightarrow} c_{n}$ в $\mathscr{C}$ категория

$$
\mathscr{D}\left(c_{0} \stackrel{f_{1}}{\longrightarrow} \cdots \stackrel{f_{n}}{\longrightarrow} c_{n}\right)=\left\{d_{0} \rightarrow \cdots \rightarrow d_{n} \mid F\left(d_{i-1} \rightarrow d_{i}\right)=f_{i}\right\}
$$

имеет стягиваемый нерв.

ЗАмечАниЕ 13. Если вдобавок $\mathscr{D} \rightarrow \mathscr{C}$ - оп-расслоение, то введенное понятие резольвенты совпадает с описанным нами ранее в [15], [16]. В этом случае функтор $\mathscr{D}\left(c_{0} \rightarrow\right.$ $\left.\cdots \rightarrow c_{n}\right) \rightarrow \mathscr{D}\left(c_{0}\right)$ допускает сопряженный, и по известной теореме Квиллена [18] это дает гомотопическую эквивалентность нервов $\mathscr{D}\left(c_{0} \rightarrow \cdots \rightarrow c_{n}\right)$ и $\mathscr{D}\left(c_{0}\right)$.

Если дан функтор $F: \mathscr{D} \rightarrow \mathscr{C}$, то по модельному оп-расслоению $\mathscr{E} \rightarrow \mathscr{C}$ можно индуцировать (посредством взятия обратного образа) модельное оп-расслоение $\mathscr{E} \rightarrow \mathscr{D}$ над $\mathscr{D}$. На предсечениях возникает функтор $\mathbb{F}^{*}: \operatorname{PSect}(\mathscr{C}, \mathscr{E}) \rightarrow \operatorname{PSect}(\mathscr{D}, \mathscr{E})$, он ограничивается на гомотопические категории производных сечений и дает функтор

$$
\mathrm{hF}^{*}: \operatorname{HoDSect}(\mathscr{C}, \mathscr{E}) \rightarrow \operatorname{HoDSect}(\mathscr{D}, \mathscr{E}) .
$$

Теорема 14. Пусть $F: \mathscr{D} \rightarrow \mathscr{C}$ - резольвента в смысле определения 12 . Тогда существует функтор $\mathrm{h} \mathbb{F}_{!}: \operatorname{Ho} \operatorname{DSect}(\mathscr{D}, \mathscr{E}) \rightarrow \operatorname{HoDSect}(\mathscr{C}, \mathscr{E})$, который сопряжен $\mathrm{hF}^{*}$ слева на гомотопических категориях. Кроме того, $\mathrm{hF}^{*}$ строго полон, а композииия $\mathrm{h} \mathbb{F} ! \mathrm{hF}^{*}$ изоморфна тождественному функтору.

В качестве приложения этого результата мы предлагаем доказательство гипотезы Делиня в формализме Сигала. Для этого определим следующие категории В и Т. Предварительно заметим, что всякий морфизм отмеченных множеств $f: T \rightarrow U$ индуцирует морфизм $\bar{f}: T^{\prime} \rightarrow U \backslash u$, где $U \backslash u$ - подмножество всех неотмеченных точек $U$ и $T^{\prime}=f^{-1}(U \backslash u)$.

ОПредЕЛЕниЕ 15. Объект категории В - инъективное пунктированное отображение $i_{T}:(T, t) \hookrightarrow\left(S^{2}, s\right)$ отмеченного конечного множества $T$ в двумерную сферу $S^{2}$ с фиксированной точкой $s$. Ограничение на подмножество $T^{\prime} \subset T$, не содержащее отмеченную точку, естественно индуцирует инъективное отображение $j_{T^{\prime}}: T^{\prime} \rightarrow D^{2}$ в двумерный диск. Морфизм между двумя объектами $i_{T}, j_{U}$, состоит из морфизма $f: T \rightarrow U$ отмеченных множеств, который индуцирует $\bar{f}: T^{\prime} \rightarrow U \backslash u$, и пути между $j_{T^{\prime}}$ и $j_{U \backslash u}$ в стратифицированном фундаментальном группоиде [19] $\Pi_{1}^{\text {strat }}\left(\operatorname{Conf}\left(D^{2},\left|T^{\prime}\right|\right)\right)$ пространства $\operatorname{Conf}\left(D^{2},\left|T^{\prime}\right|\right)$ конфигураций $\left|T^{\prime}\right|$ точек на двумерном диске.

ЗАмечание 16 . Пусть $\mathscr{M}-\mathrm{CMK}$, тогда естественный функтор В $\rightarrow \Gamma$ индуцирует оп-расслоение $\mathscr{M}^{\otimes} \rightarrow$ В. Производные сечения этого оп-расслоения естественным образом отвечают факторизационным алгебрам в смысле [20]. С точностью до квазиизоморфизма, они также соответствуют $\mathbb{E}_{2}$-алгебрам в смысле [9]-[11].

ОПредЕлЕниЕ 17. Объектами категории Т являются планарные деревья с корнем, вершинами, отмеченными конечным множеством $S$, и стабильными неотмеченными вершинами [21]. Морфизмы между деревьями даны отображением конечных множеств, стягиванием ребер и стабилизацией. 
Существует также категория $\widetilde{\mathrm{T}}$, объекты которой такие же, как в Т, вложенные при этом в диск. Корни всех деревьев при этом переходят в фиксированную точку на границе. Естественный функтор $\widetilde{\mathrm{T}} \rightarrow \mathrm{T}$ является эквивалентностью категорий, а забывание дерева (остаются отмеченные вершины) индуцирует функтор $\widetilde{\mathrm{T}} \rightarrow$ В. Обращение эквивалентности задает функтор $F: \mathrm{T} \rightarrow \mathrm{B}$.

Теорема 18 ([21], см. также [22]). Функтор $F: \mathrm{T} \rightarrow$ В является резолъвентой в смьсле определения 12.

Обозначим через $\mathbf{D V e c t}_{k}^{\otimes} \rightarrow$ Т оп-расслоение, индуцированное с $\mathbf{D V e c t}_{k}^{\otimes} \rightarrow \Gamma$ по функтору $\mathrm{T} \rightarrow \mathrm{B} \rightarrow \Gamma$. Пусть дана $d g k$-алгебра $A$, и обозначим также $A^{\text {op }} d g$-алгебру с обратным умножением, а за $A^{*}$ - двойственное $k$-векторное пространство к $A$. Тогда можно определить [23] Т-сечение этого оп-расслоения, которое соответствует когомологическому комплексу $C H(A, A)$ Хохшильда алгебры $A$, следующим образом.

Существует опрасслоение $\operatorname{Bimod}_{A}^{\otimes} \rightarrow \mathrm{T}$, слой которого над деревом $t \in \mathrm{T}$ есть

$$
\operatorname{Bimod}_{A}^{\otimes}(t)=\prod_{v} A^{\otimes i n(v)} \otimes A^{\mathrm{op}}-\operatorname{Bimod},
$$

то есть произведение, взятое по всем вершинам $v$, кроме корня, категорий $A^{\otimes \operatorname{in}(v)} \otimes A^{\text {oр }-б и-~}$ модулей, где in $(v)$ обозначает число ребер, входящих в $v$. Существует функтор

$$
L: A^{\otimes n} \otimes A^{\text {op }} \text {-Bimod } \rightarrow A \text {-Bimod } \quad \text { со значением } \quad L(M)=M \otimes_{A^{\otimes n} \otimes A^{o \otimes n}} A^{\otimes n} .
$$

ПреДЛОЖениЕ 19. Для функтора L существует правый сопряженный

$$
R: A \text {-Bimod } \rightarrow A^{\otimes n} \otimes A^{\mathrm{op}} \text {-Bimod, } \quad R(N)=A^{* \otimes n} \otimes N,
$$

который точен и, более того,

$$
H H^{*}\left(A^{\otimes n} \otimes A^{\mathrm{op}}, R(M)\right)=H H^{*}(A, M) .
$$

Функтор $L$ можно продолжить до отображения оп-расслоений

$$
L: \operatorname{Bimod}_{A}^{\otimes} \rightarrow A \text {-Bimod } \times \mathrm{T},
$$

где $A$-Bimod $\times$ T $\rightarrow$ T есть постоянное оп-расслоение.

Teорема 20. Пусть $\mathscr{E} \rightarrow \mathscr{C}$ и $\mathscr{F} \rightarrow \mathscr{C}-$ два модельных оп-расслоения и $G: \mathscr{E} \rightarrow \mathscr{F}$ функтор, коммутирующий с проекциями в $\mathscr{C}$, так что послойно $G(c): \mathscr{E}(c) \rightarrow \mathscr{F}(c)$ является левым квилленовым функтором с правым сопряженным $H(c)$. Тогда естественно индуиированный функтор $G: \operatorname{DSect}(\mathscr{C}, \mathscr{E}) \rightarrow \operatorname{DSect}(\mathscr{C}, \mathscr{F})$ имеет правый сопряженный $h H$ на гомотопических категориях.

Таким образом, из сечения $A: \mathrm{T} \rightarrow A$-Bimod $\times \mathrm{T}, A(t)=(A, t)$, мы получаем сечение $R(A): \mathrm{T} \rightarrow \operatorname{Bimod}_{A}^{\otimes}$. Сопоставление

$$
M \in \operatorname{Bimod}_{A}^{\otimes}(t) \cong A^{\otimes \text { out }(t)} \otimes A^{\text {op }}-\operatorname{Bimod} \mapsto C H\left(A^{\otimes \text { out }(t)} \otimes A^{\text {op }}, M\right)
$$

задает морфизм Т-оп-расслоений $C H: \operatorname{Bimod}_{A}^{\otimes} \rightarrow \operatorname{DVect}_{k}^{\otimes}$, и нужное нам сечение получается как $C H(R(A))$. Применение теорем 14 и 18 к полученному Т-сечению дает нам производное В-сечение, которое описывает когомологический комплекс Хохшильда как $\mathbb{E}_{2}$-алгебру в сигаловом формализме. 


\section{СПИСОК ЦИТИРОВАННОЙ ЛИТЕРАТУРЫ}

[1] J. F. Adams, Infinite Loop Spaces, Ann. of Math. Stud., 90, Princeton Univ. Press, Princeton, NJ, 1978. [2] J. P. May, The Geometry of Iterated Loop Spaces, Lectures Notes in Math., 271, Springer-Verlag, Berlin, 1972. [3] G. Segal, Categories and Cohomology Theories, Topology, 13, 1974. [4] S. Mac Lane, Categories for The Working Mathematician, Graduate Texts in Math., 5, Springer-Verlag, New York, 1998. [5] T. Leinster, Higher Operads, Higher Categories, London Math. Soc. Lecture Note Ser., 298, Cambridge Univ. Press, Cambridge, 2004. [6] D. Quillen, Higher K-Theories, Lecture Notes in Math., 341, Springer-Verlag, Berlin, 1973, 85-147. [7] W. G. Dwyer, Ph. S. Hirschhorn, D. M. Kan, J. H. Smith, Homotopy Limit Functors on Model Categories and Homotopical Categories, Math. Surveys Monogr., 113, Amer. Math. Soc., Providence, RI, 2004. [8] M. Hovey, Model Categories, Math. Surveys Monogr., 63, Amer. Math. Soc., Providence, RI, 1999. [9] J. E. McClure, J. H. Smith, Recent Progress in Homotopy Theory, Contemp. Math., 293, Amer. Math. Soc., Providence, RI, 2002, 153-193.. [10] Dmitry E. Tamarkin, Another Proof of M. Kontsevich Formality Theorem, 1998, arXiv: math.QA/9803025. [11] K. Costello, Adv. Math., 210:1 (2007), 165-214. [12] C. Barwick, From Operator Categories to Topological Operads, 2013, arXiv: 1302.5756. [13] Revêtements étales et groupe fondamental, Lecture Notes in Math., 224, Springer-Verlag, Berlin, 1971. [14] J. Lurie, Higher Algebra, 2016, http://www.math.harvard.edu/ lurie/papers/HA.pdf. [15] E. Balzin, Derived Sections of Grothendieck Fibrations and the Problems of Homotopical Algebra, 2015, arXiv: 1410.3387. [16] Э. Бальзин, УМН, 69:5 (419) (2014), 159-160. [17] A. K. Bousfield, D. M. Kan, Homotopy Limits, Completions and Localizations, Lecture Notes in Math., 304, Springer-Verlag, Berlin, 1972. [18] D. G. Quillen, Homotopical Algebra, Lecture Notes in Math., 43, Springer-Verlag, Berlin, 1967. [19] T. Woolf, The Fundamental Category of a Stratified Space, 2013, arXiv: 0811.2580. [20] A. Beilinson, V. Drinfeld, Chiral Algebras, Amer. Math. Soc. Colloq. Publ., 51, Amer. Math. Soc., Providence, RI, 2004. [21] M. Kontsevich, Y. Soibelman, Homological Mirror Symmetry, Lecture Notes in Phys., 757, Springer-Verlag, Berlin, 2008, 1-67. [22] D. Kaledin, Homological Methods in Non-commutative Geometry, Lecture Notes, University of Tokyo, Tokyo, 2008. [23] D. Kaledin, Non-Commutative Geometry from the Homological Point of View, Lecture Notes, KIAS, Seoul, 2009.

Э. Р. Бальзин

Национальный исследовательский университет

"Высшая школа экономики", г. Москва;

Laboratoire de Mathématiques

Jean Alexandre Dieudonné,

Université de Nice Sophia Antipolis, Франция

E-mail: eduard.r.balzin@gmail.com,

erbalzin@edu.hse.ru, balzin@unice.fr
Поступило 25.02 .2016 